\title{
Combination Therapy with Systemic Steroids, an Antiviral Agent, Anticoagulants, and Stellate Ganglion Block for Treatment of Sudden Sensorineural Hearing Loss
}

\author{
Kye Hoon Park, Chi-Kyou Lee, Jong Dae Lee, Moo Kyun Park and Byung Don Lee \\ Department of Otorhinolaryngology-Head and Neck Surgery, Soonchunhyang University College of Medicine, Cheonan, Korea
}

Received August 10,2012

Revised August 22, 2012

Accepted September 4, 2012

Address for correspondence Kye Hoon Park, MD

Department of Otorhinolaryngology-

Head and Neck Surgery,

Soonchunhyang University

College of Medicine,

31 Soonchunhyang 6-gil, Dongnam-gu,

Cheonan 330-721, Korea

Tel $+82-41-570-3832$

Fax $+82-41-579-9022$

E-mail earpark@gmail.com
Background and Objectives: Sudden sensorineural hearing loss (SSNHL) is commonly defined as a loss of at least $30 \mathrm{~dB}$ in three contiguous frequencies occurring within 3 days. Systemic steroid administration has become the most widely accepted treatment option for SSNHL. Since viral infection and vascular compromise are considered specific causes of SSNHL, antiviral agents, anticoagulants, and stellate ganglion block have been used for its treatment, although the evidence of their effectiveness is weak. The present study evaluated the hearing recovery rate in the combination therapy group (systemic steroids, antiviral agent, anticoagulants, and stellate ganglion block) in comparison with patients treated with systemic steroids alone. Subjects and Methods: A total of 85 patients diagnosed with SSNHL were treated with combination therapy (group A, 46 patients) or systemic steroids only (group B, 39 patients). Hearing improvement was defined as a hearing gain of more than slight improvement using Siegel's criteria. All patients were treated with a 10-day course of systemic steroids (10-mg dexamethasone for 5 days, followed by tapering for 5 days). Acyclovir, heparin, and stellate ganglion block were included in the group A treatment regimen. Results: The overall rate of hearing improvement was $60.9 \%$ (28/46 patients) in group A, which was significantly higher than that (38.5\%, 15/39 patients) in group B. The distribution of prognostic factors was not significantly different between the two groups with the exception of the degree of initial hearing loss, which was more severe in group A. Upon analysis according to prognostic factors, group $\mathrm{A}$ showed a better hearing improvement recovery rate than group $\mathrm{B}$ in patients with hearing loss $>70 \mathrm{~dB}$, age $>41$ years, dizziness, and early treatment ( $<1$ week). Conclusions: Thus SSNHL patients treated with combination therapy have a higher likelihood of hearing improvement than those treated with systemic steroids alone.

Korean J Audiol 2012;16:71-74

KEY WORDS: Idiopathic sudden sensorineural hearing loss · Hearing recovery · Steroid Combination therapy.

\section{Introduction}

Sudden sensorineural hearing loss (SSNHL) is commonly defined as a loss of at least $30 \mathrm{~dB}$ in three contiguous frequencies occurring within 3 days. ${ }^{1)}$ Lack of knowledge of the specific causes of idiopathic SSNHL limits our ability to implement effective treatment. Although it is possible that a number of different pathological processes result in sudden hearing loss, it is widely believed that viral infection and vascular occlusion account for the majority of these cases. ${ }^{2-4)}$ Systemic steroid administration is the most widely accepted treatment option for SSNHL. ${ }^{5)}$ Since viral infection and vascular compromise are considered specific causes of SSNHL, antiviral agents, anticoagulants, and stellate ganglion block have been used for its treatment, although the evidence of their effectiveness is weak. ${ }^{2-4,6)}$

In our hospital, one author used a combination therapy consisting of systemic steroids, an antiviral agent, anticoagulants, and stellate ganglion block for the treatment of SSNHL, while two authors employed systemic steroids only to determine whether there is a difference in hearing recovery rate between the two treatment options. 
The present study evaluated the hearing recovery rate in the combination therapy group (systemic steroids, an antiviral agent, anticoagulants, and stellate ganglion block) in comparison with the group treated with systemic steroids alone.

\section{Subjects and Methods}

Clinical records of subjects with unilateral SSNHL were retrospectively reviewed. A total of 85 patients diagnosed with SSNHL were treated with either combination therapy (group A, 46 patients) or systemic steroids only (group B, 39 patients). The study population consisted of 43 females and 42 males ranging in age from 14 to 77 years, with a mean age of 49.2 years. The left ear was involved in 39 patients and the

Table 1. Siegel's hearing recovery criteria

\begin{tabular}{lc}
\hline \multicolumn{1}{c}{ Type } & Hearing recovery \\
\hline I. Complete recovery & Final hearing better than $25 \mathrm{~dB}$ \\
II. Partial recovery & More than $15 \mathrm{~dB}$ gain, final hearing \\
& $25-45 \mathrm{~dB}$ \\
III. Slight Improvement & More than $15 \mathrm{~dB}$ gain, final hearing \\
& poorer than $45 \mathrm{~dB}$
\end{tabular}

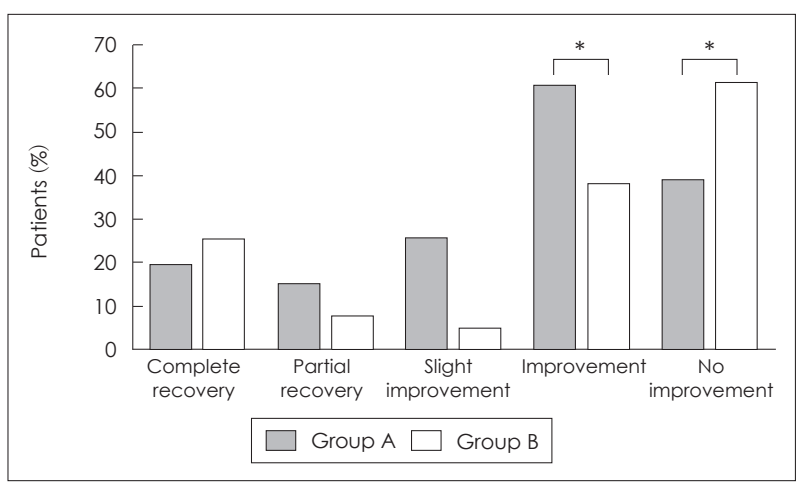

Fig. 1. Hearing recovery rate after combined therapy (group $A$ ) and systemic steroids only (group B). Improvement=complete recovery+partial recovery+slight improvement. ${ }^{*} p<0.05, \chi^{2}$ test. right in 46. The distribution of sex and age was not different between the two groups.

All patients were treated with a 10-day course of systemic steroids (10 $\mathrm{mg}$ of dexamethasone for 5 days, followed by tapering for 5 days). The treatment regimen of group A included acyclovir (250 mg, 3 times daily, 5 days), heparin (low molecular weight heparin $10000 \mathrm{U}$ daily, 10 days), and stellate ganglion block (daily by anesthesiologist, 5-10 days). Auditory function was determined by pure tone audiometry, and mean hearing levels were expressed as the average of hearing thresholds at 500, 1000, 2000, and $3000 \mathrm{~Hz}$. Auditory measurements were performed before and 2 months after treatment using Siegel's hearing improvement criteria (Table 1). Hearing improvement was defined as a hearing gain of more than slight improvement using Siegel's criteria.

The following prognostic factors were taken into consideration in the analysis of hearing recovery rates: 1) initial hearing level (>70 dB HL), 2) audiogram shape (ascending, descending, and flat type), 3) treatment onset (within 7 days), 4) age (>40 years), and 5) presence of dizziness. Statistical evaluations were performed using the chi-square test, Fisher's exact test, and logistic regression.

\section{Results}

The overall rate of hearing improvement was $60.9 \%$ (28/46 patients) in group A, which was significantly higher than that $(38.5 \%, 15 / 39$ patients $)$ in group $\mathrm{B}\left(p=0.039, \chi^{2}\right.$ test)(Fig. 1). Group A included nine patients (19.6\%) with complete hearing recovery, seven $(15.2 \%)$ with partial recovery, and $12(26.1 \%)$ with slight improvement. group B included 10 patients $(25.6 \%)$ with complete hearing recovery, three $(7.7 \%)$ with partial recovery, and two $(5.1 \%)$ with slight improvement (Fig. 1).

The distribution of prognostic factors was not significantly different between the two groups, with the exception of degree

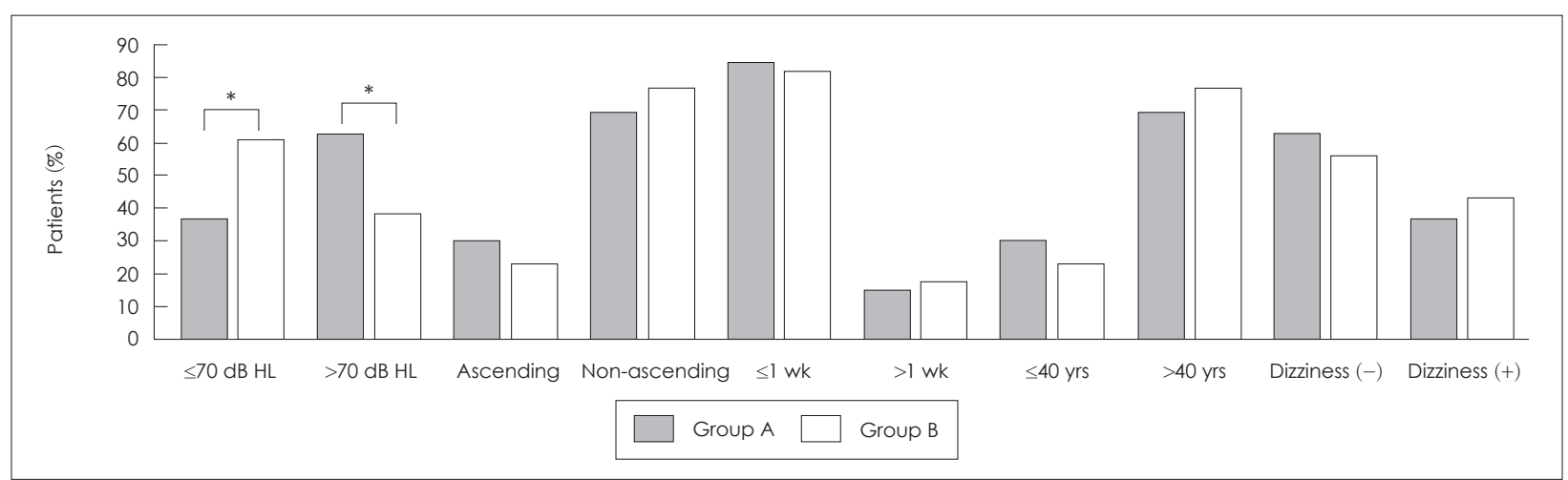

Fig. 2. The distributions of prognostic factors were not significantly different between groups $A$ and $B$, with the exception of degree of initial hearing loss, which was more severe in group $A .{ }^{*} p<0.05, \chi^{2}$ test. 
of initial hearing loss, which was more severe in group A (Fig. 2).

Hearing improvement was analyzed according to the prognostic factors, and the results indicated a better recovery rate in group A compared to group B among patients with hearing loss $>70 \mathrm{~dB}$, age $>41$ years, dizziness, and early start of treatment $(<1$ week $)\left(p<0.05, \chi^{2}\right.$ test, Fisher's exact test)(Table 2) (Fig. 3).

Multivariate analysis of potential prognostic factors, including treatment option (group A or B), indicated a significant association between an ascending audiogram shape and good clinical outcome. The level of hearing loss, the pres ence of diz- ziness, delay of treatment, age $>40$ years, and treatment options showed no associations with hearing recovery (Table 3 ).

\section{Discussion}

The current standard treatment for SSNHL is a tapered course of systemic steroids. ${ }^{7)}$ However, data to support this recommendation are limited. A 2006 Cochrane database review concluded that the value of steroids in the treatment of SSNHL remains unclear. ${ }^{8)}$ The mechanism of action of steroids in the inner ear is incompletely understood. The actions

Table 2. Hearing recovery rate according to prognostic factors in groups $A$ and $B$

\begin{tabular}{|c|c|c|c|}
\hline \multirow{2}{*}{\multicolumn{2}{|c|}{ Prognostic factor }} & \multicolumn{2}{|c|}{ Recovery rate } \\
\hline & & Group A & Group B \\
\hline \multirow[t]{2}{*}{ Initial hearing level } & $\leq 70 \mathrm{~dB} \mathrm{HL}$ & $41.2 \%$ (7/17 patients) & $50.0 \%$ (12/24 patients) \\
\hline & $>70 \mathrm{~dB} \mathrm{HL} *$ & $72.4 \%(21 / 29$ patients $)$ & $20 \%$ (3/15 patients) \\
\hline \multirow[t]{2}{*}{ Audiogram shape } & Ascending type & $64.7 \%(11 / 14$ patients $)$ & $66.7 \%$ (6/9 patients) \\
\hline & Non-ascending type & $53.1 \%$ ( $17 / 32$ patients $)$ & $30 \%$ (9/30 patients) \\
\hline \multirow[t]{2}{*}{ Treatment onset } & $\leq 1 w^{*}$ & $66.7 \%$ (26/39 patients) & $40.6 \%$ (13/32 patients) \\
\hline & $>1 \mathrm{wk}$ & $28.6 \%$ (2/7 patients) & $28.6 \%$ (2/7 patients) \\
\hline \multirow[t]{2}{*}{ Age } & $\leq 40$ yrs & $64.3 \%$ (9/14 patients) & $55.6 \%$ (5/9 patients) \\
\hline & $>40$ yrs* & $59.4 \%$ (19/32 patients) & $33.3 \%$ (10/30 patients) \\
\hline \multirow[t]{2}{*}{ Presence of dizziness } & Dizziness $(-)$ & $62.1 \%$ (18/29 patients) & $54.5 \%$ (12/22 patients) \\
\hline & Dizziness $(+)^{*}$ & $58.8 \%$ (10/17 patients) & $17.6 \%$ (3/17 patients) \\
\hline
\end{tabular}

$* p<0.05, \chi^{2}$ test, Fisher's exact test

Table 3. Multivariate analysis of the significant prognostic factors

\begin{tabular}{|c|c|c|c|c|c|}
\hline Variables & $\beta$ & SE & Sig. & Odds ratio & $95 \% \mathrm{Cl}$ \\
\hline Combination therapy & 0.698 & 0.513 & 0.174 & 2.009 & $0.735-5.496$ \\
\hline Age $\leq 40$ yrs & 0.663 & 0.565 & 0.240 & 1.941 & $0.642-5.869$ \\
\hline Treatment onset $\leq 1 \mathrm{wk}$ & 1.291 & 0.741 & 0.081 & 3.637 & $0.852-15.530$ \\
\hline Absence of dizziness & 0.738 & 0.504 & 0.143 & 2.091 & $0.779-5.615$ \\
\hline Ascending shape audiogram & 1.672 & 0.658 & $0.011^{*}$ & 5.320 & $1.465-19.325$ \\
\hline Initial hearing level $\leq 70 \mathrm{~dB} H \mathrm{H}$ & -0.510 & 0.576 & 0.376 & 0.600 & $0.194-1.856$ \\
\hline
\end{tabular}

$* p<0.05$. $\beta$ : beta-coefficient, SE: standard error, $\mathrm{Cl}$ : confidence interval

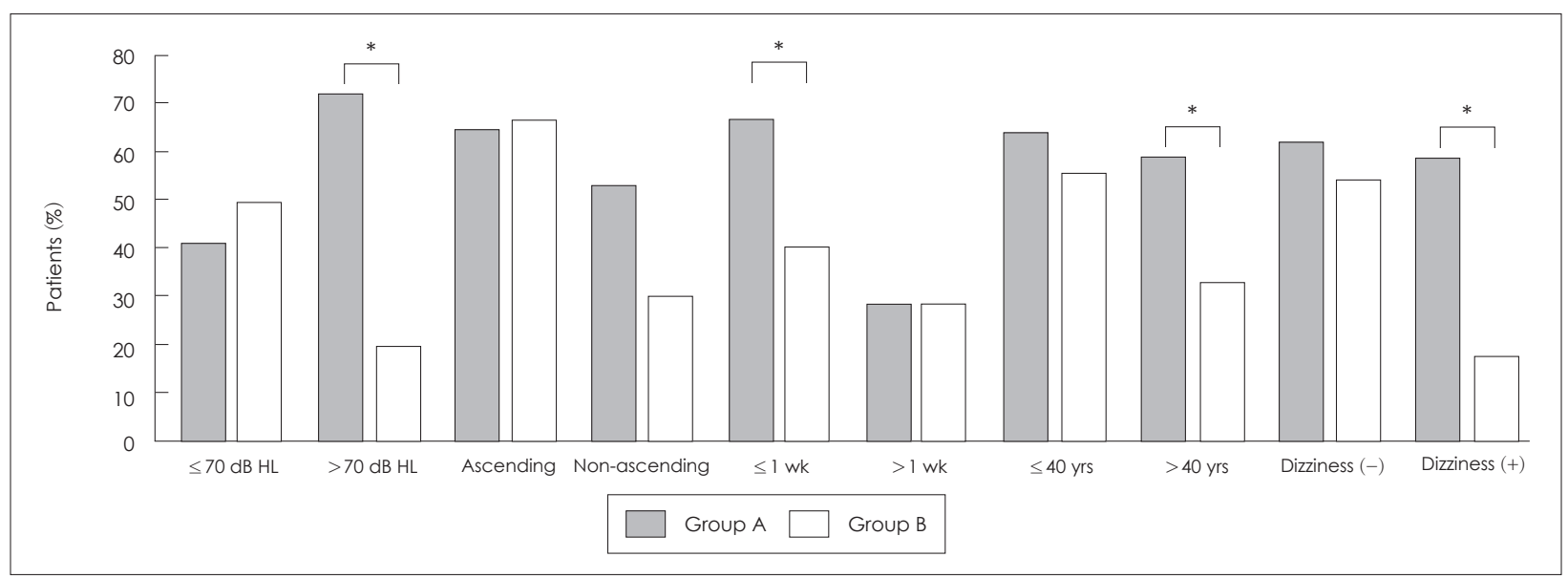

Fig. 3. Hearing recovery rate according to prognostic factors in groups A and B. * $p<0.05, \chi^{2}$ test, Fisher's exact test. 
attributed to steroids in the inner ear include ion homeostasis, antioxidant activity, inhibition of apoptosis, downregulation of local proinflammatory cytokines, and promotion of cochlear blood flow.

The potential success of antiviral medication for treatment of SSNHL is based on the suggestion that many cases of such hearing impairment have a viral etiology. ${ }^{3)}$ Herpes virus may be involved in the etiology of SSNHL by one of several mechanisms, including labyrinthitis secondary to viremia, labyrinthitis or neuritis secondary to meningitis, cranial neuropathy, reactivation of a latent ganglion cell infection, or alteration of the immune response. ${ }^{9)}$ It is possible that the viral damage to the ear is completed within a relatively short time and that inflammatory and other effects of the virus are most effectively treated thereafter with steroids. ${ }^{3)}$ This may explain the observed better recovery rate in group A than group B among patients treated within 1 week in the present study.

The use of heparin and stellate ganglion block is based on the suggestion that impairments in microcirculation of the inner ear may be involved in the pathophysiology of SSNHL. ${ }^{2,4,6)}$ The blood flow disturbance theory, including thromboembolism or vasospasm, has been proposed based on the etiology of SSNHL, because the blood supply within the inner ear is its termination and intraosseous position and symptoms of SSNHL develop abruptly. ${ }^{10,11)}$ Heparin as a treatment for SSNHL was described by Fisch, et al. ${ }^{2)}$ and it has an anticoagulant effect on intracranial blood vessels. However, Mattox and Simmons ${ }^{12)}$ reported no significant difference between patients with and without general heparin therapy. Since the introduction of low-molecular-weight heparin in the early 1990 s, its use and indications have been greatly expanded. ${ }^{13)}$ Low-molecular-weight heparin can prevent venous thrombosis and shows greatly reduced side reactions compared with general heparin. Yue, et al. ${ }^{4)}$ suggested that hearing improvement is significantly greater in patients treated with low-molecular weight heparin than in those treated with the commonly used therapeutic regimen alone.

Stellate ganglion block induces an increased blood supply via sympathetic blockade and vasodilation. ${ }^{14)}$ The eventual involvement of internal ear circulatory disturbance causes metabolic disturbance of the inner ear cells, decreases in oxidation of metabolic substrates, and edema, resulting in irreversible changes. The resorption of edema is promoted by arterial relaxation from sympathetic blockade. ${ }^{6,15)}$ Takinami ${ }^{15)}$ reported that stellate ganglion block favorably affected outcomes in patients with unilateral SSNHL given a prior or concomitant course of systemic corticosteroids.

The combination therapy showed a beneficial effect in the present study; however, the analysis was performed in a retrospective manner. Especially in patients with poor prognosis, the combined therapy resulted in a higher recovery rate than steroids alone, although the complete recovery rate was not different between the two treatment methods. The efficacy of combination therapy cannot be established definitively in a retrospective analysis with a small sample size. Therefore, our results indicate only a trend toward a beneficial effect of combination therapy as a component of SSNHL treatment.

\section{Conclusion}

The results of this study suggest that SSNHL patients treated with the combination therapy have a higher likelihood of hearing improvement than those treated with systemic steroids alone.

\section{REFERENCES}

1) Wilson WR. Why treat sudden hearing loss. Am J Otol 1984;5:481-3.

2) Fisch U, Nagahara K, Pollak A. Sudden hearing loss: circulatory. Am J Otol 1984;5:488-91.

3) Tucci DL, Farmer JC Jr, Kitch RD, Witsell DL. Treatment of sudden sensorineural hearing loss with systemic steroids and valacyclovir. Otol Neurotol 2002;23:301-8

4) Yue WL, Li P, Qi PY, Li HJ, Zhou H. Role of low-molecular-weight heparins in the treatment of sudden hearing loss. Am J Otolaryngol 2003;24:328-33.

5) O'Malley MR, Haynes DS. Sudden hearing loss. Otolaryngol Clin North Am 2008;41:633-49.

6) Cook TG, de Sanctis CA, Plaza JA, Sawyer DD. Stellate ganglion block for sudden profound hearing loss. Anesthesiology 1981;54:421-3.

7) Westerlaken BO, de Kleine E, van der Laan B, Albers F. The treatment of idiopathic sudden sensorineural hearing loss using pulse therapy: a prospective, randomized, double-blind clinical trial. Laryngoscope 2007;117:684-90.

8) Wei BP, Mubiru S, O'Leary S. Steroids for idiopathic sudden sensorineural hearing loss. Cochrane Database Syst Rev 2006:CD003998.

9) Wilson WR. The relationship of the herpesvirus family to sudden hearing loss: a prospective clinical study and literature review. Laryngoscope 1986;96:870-7.

10) García Callejo FJ, Martínez Beneyto MP, Platero Zamarreño A, Marco Sanz M, Fernández Julián EN, Marco Algarra J. [Non-interventional study on blood filterability changes in the clinical onset of sensorineural sudden deafness]. Acta Otorrinolaringol Esp 2001;52:55664.

11) Nomura Y. Diagnostic criteria for sudden deafness, mumps deafness and perilymphatic fistula. Acta Otolaryngol Suppl 1988;456:7-8.

12) Mattox DE, Simmons FB. Natural history of sudden sensorineural hearing loss. Ann Otol Rhinol Laryngol 1977;86(4 Pt 1):463-80.

13) McKay GA, Paterson KR. Low molecular weight heparins--a safer option than unfractionated heparin? Adverse Drug React Toxicol Rev 2001;20:256-76.

14) Haug O, Draper WL, Haug SA. Stellate ganglion blocks for idiopathic sensorineural hearing loss. Arch Otolaryngol 1976;102:5-8.

15) Takinami Y. Evaluation of effectiveness of stellate ganglion block (SGB) treatment of sudden hearing loss. Acta Otolaryngol 2012;132: 33-8. 